Pract. oto-rhino-laryng. 1970;32:128

\title{
Varia
}

\section{Fellowship in Otorhinolaryngology}

Fellowships are available in otorhinolaryngology for postresidency training in otology, laryngology and communicative disorders. The fellowships are for 1-3 years and can either be completely clinical or can be combined with laboratory work. For further information, contact Dr. R. J. Ruben, Director of the Division of Otolaryngology, Department of Surgery, Albert Einstein College of Medicine, 1300 Morris Park Avenue, New York, N.Y. 10461 (USA). Internationales Symposion für endolaryngologische Mikrochirurgie Vom 22. bis 27. Juni 1970 findet in Barcelona ein Internationales Symposion für endolaryngologische Mikrochirurgie statt. Weitere Auskünfte erteilt das Servicio ORL, Hospital de la Cruz Roja, Dos de Mayo, 301, Barcelona (Spanien). 54. Versammlung der Vereinigung Südwestdeutscher HNO-Ärzte Die 54. Versammlung der Vereinigung Südwestdeutscher HNO-Ärzte wird am 25. und 26. September 1970 in Konstanz stattfinden. Verhandlungsthemen: Möglichkeiten zur Rationalisierung der Arbeit des HNO-Arztes in Klinik und Praxis. Anfragen sind zu richten an Prof. Dr. J. Matzker, HNO-Klinik, Ostmerhei-merstrasse 200, D-5 Köln-Merheim (Deutschland). 